Research Article

\title{
Ocular Surface Disease Index and Ocular Thermography in Keratoconus Patients
}

\author{
Orsolya Németh $\mathbb{D}^{1,2,3}$ Achim Langenbucher, ${ }^{4}$ Timo Eppig, ${ }^{4}$ Sabine Lepper, ${ }^{1}$ \\ Georgia Milioti, ${ }^{1}$ Aladin Abdin, ${ }^{1}$ Zoltán Zsolt Nagy, ${ }^{3}$ Berthold Seitz, ${ }^{1}$ and Nóra Szentmáry ${ }^{1,3}$ \\ ${ }^{1}$ Department of Ophthalmology, Saarland University Medical Center, Homburg, Saarland 66424, Germany \\ ${ }^{2}$ Department of Ophthalmology, Markusovszky University Teaching Hospital, Szombathely 9700, Hungary \\ ${ }^{3}$ Department of Ophthalmology, Semmelweis University, Budapest 1085, Hungary \\ ${ }^{4}$ Experimental Ophthalmology, Saarland University, Homburg, Saarland 66424, Germany
}

Correspondence should be addressed to Orsolya Németh; nemeth.orsolya22@gmail.com

Received 27 October 2019; Revised 4 January 2020; Accepted 4 February 2020; Published 24 February 2020

Academic Editor: Antonio Queiros

Copyright (c) 2020 Orsolya Németh et al. This is an open access article distributed under the Creative Commons Attribution License, which permits unrestricted use, distribution, and reproduction in any medium, provided the original work is properly cited.

\begin{abstract}
Purpose. Keratoconus (KC) has been defined as a "noninflammatory" corneal disease, but recent studies have noted a potential inflammatory origin. We analysed the Ocular Surface Disease Index (OSDI) and ocular surface temperature (OST) in KC patients compared to controls. Patients and Methods. A total of 179 eyes in 90 patients with KC (topographic keratoconus classification $0-1$ to 4 , age $36.1 \pm 12.5$ years, $65.9 \%$ males) and 82 eyes in 41 controls (age $36.4 \pm 12.8$ years, $47.6 \%$ males) were examined. The participants completed the OSDI questionnaire and underwent corneal topography, tomography, and thermography. Additional outcome measures were vision- and discomfort-related OSDI subscores and mean OST at the corneal centre during 10 seconds of sustained eye opening after blinking. Results. The OSDI score ( $31.4 \pm 22.4$ vs. $17.5 \pm 17.9)$ and vision- (17.7 \pm 14.6 vs. $10.5 \pm 13.2)$ and discomfort-related (14.3 \pm 10.7 vs. $9.4 \pm 10.5)$ OSDI subscores were significantly higher in KC patients than in controls $(p<0.001)$. We found no significant difference in the central corneal OST $\left(34.2 \pm 0.6^{\circ} \mathrm{C}\right.$ vs. $34.2 \pm 0.7^{\circ} \mathrm{C}$; $\left.p=0.41\right)$ between the two groups $(p \geq 0.22)$. The OSDI score and subscores poorly to fairly correlated with the surface asymmetry index (SAI) and surface regularity index (SRI; $r>0.174, p<0.005)$, but did not correlate with the central corneal OST $(r<0.001)$. OST also did not correlate with the SAI, SRI, and central corneal thickness $(r \geq-0.086)$. Conclusion. KC patients had increased OSDI scores and vision- and discomfort-related OSDI subscores without an increase in the OST compared to a normal population. OSDI score/subscores weakly correlate with SAI and SRI but do not correlate with OST in KC patients or controls. Vision- and discomfort-related symptoms of $\mathrm{KC}$ have to be managed in parallel in ophthalmological practice, but the necessity of antiinflammatory treatment cannot be verified through ocular thermography.
\end{abstract}

\section{Introduction}

Thermography is used in many fields of medicine, including angiology [1], oncology [2], and rheumatology [3]. Since Mapstone [4-6] introduced infrared thermography of the ocular surface, the method has become widely used. In ophthalmology, ocular surface temperature (OST) has been investigated in ocular inflammation [7], tear film abnormalities [8], analysis of bleb function after glaucoma surgery [9] and after corneal refractive surgery [10] and cataract surgery [11], and in the evaluation of ocular blood flow [12].
OST may be influenced by environmental and ocular factors. In the environment, changes in the ambient temperature may influence OST, but it reaches a plateau of $36^{\circ} \mathrm{C}$ at an ambient temperature of $40^{\circ} \mathrm{C}[13,14]$. Wind, air conditioning, or any kind of air flow affects OST through increased evaporation [15], and lower air humidity increases lacrimal evaporation [16]. Blinking interrupts corneal exposure to the environment and redistributes the tear film and its temperature [17].

OST also depends on ocular factors, such as the quality and quantity of the tear film and heat conduction and 
convention of the aqueous humour, which is determined mainly through blood flow in the ciliary body and through retrobulbar haemodynamics [18]. As the cornea is an avascular tissue, the central corneal temperature is mainly influenced by tear film evaporation and heat convection and conduction of the aqueous humour [18]. Nevertheless, the temperature of the peripheral cornea may also be influenced by blood flow in the perilimbal vessels [12].

Keratoconus (KC) is a bilateral, asymmetric condition characterized by progressive thinning and deformation of the corneal tissue, which may lead to significant visual impairment due to irregular corneal astigmatism [19]. Its prevalence is approximately $1: 2000$ in the Caucasian population, but its exact aetiology remains unknown. Although $\mathrm{KC}$ cases are sporadic, some studies have reported autosomal dominant or recessive inheritance [20]. Eye rubbing may be the most important environmental factor related to the development of KC. Therefore, patients with a history of atopy may have a higher risk of developing KC [21].

Although KC is defined as a "noninflammatory" corneal disease, several studies have reported a potential inflammatory origin. For example, proinflammatory cytokines IL6 , IL- $1 \beta$, IFN- $\gamma$, and TNF- $\alpha$ have been demonstrated in the tear film of KC patients [22-24]. In clinical studies on KC patients, ocular surface disease is characterized by worse tear quality, significantly lower break-up time (BUT), and higher fluorescein and rose bengal staining scores than the normal population [25]. A correlation between ocular surface disease and KC stage has also been verified [25]. Although some of the KC screening indices are also sensitive to dry eye syndrome [26], no interaction between measures of dry eye syndrome and topographic/tomographic changes in $\mathrm{KC}$ patients could be shown [27].

To add insight into the relationship between ocular surface disease and KC, we analysed the Ocular Surface Disease Index (OSDI) and OST in KC patients compared to controls.

\section{Patients and Methods}

All examinations were performed following the regulations of the Declaration of Helsinki. Our study was approved by the Ethics Committee of Saarland/Germany (no. 41/18), and informed consent was obtained from all participants at the Homburg Keratoconus Center [28].

Patients with the diagnosis of KC who had not had previous ocular surgery were included in the study. Additional exclusion criteria were tight palpebral fissure, rapid eye movements during the examination, diagnosis of pellucide marginal degeneration, and keratoglobus. KC was diagnosed by slitlamp examination and corneal topography (TMS-5, Tomey, Erlangen-Tennenlohe, Germany) and tomography (Pentacam, Wetzlar, Germany). Previous contact lenses were not an exclusion criterion, but patients had to be examined after at least 2 days without wearing contact lenses.

Each participant completed the Ocular Surface Disease Index (OSDI) questionnaire. A total OSDI score, vision- related subscore (derived from questions about vision and task performance), and discomfort-related subscore (derived from questions about ocular surface discomfort) were calculated for each participant as described by Mathews et al. [29]. Score ranges were designated as normal (0-12), mild (13-22), moderate (23-32), or severe (33-100) ocular surface disease.

As the patients filled out the OSDI questionnaire, the OST adapted to the ambient temperature of the standardized examination room. We then measured the OST using the TG-1000 Ocular Surface Thermographer (Tomey, Erlangen-Tennenlohe, Germany). All examinations were performed by the same examiner to eliminate interexaminer variation [30]. During the measurements, a standard environment was maintained in the examination room with an average room temperature of $23.9 \pm 1.6^{\circ} \mathrm{C}$ and humidity of $32.4 \pm 6.7 \%$. The doors and windows in the examination room were closed to minimize air flow, which has been reported to have a significant effect on the OST [15].

The OST was measured using the method described by Mori and associates: the participants blinked normally, closed both eyes for 5 seconds, and then kept their eyes open for more than 10 seconds [31]. During the examination, the participants' head was placed in a standard ophthalmic chin and head rest, and they were instructed to look straight ahead. For each measurement, a sequence of 11 OST images was taken from baseline to 10 seconds after eye opening (1 per second). With a lateral resolution of $70 \mu \mathrm{m}$ in both the horizontal and vertical directions, $320 \times 240$ data points were stored with each image. We extracted mean OST values at the corneal centre and $2 \mathrm{~mm}$ from the corneal centre nasally, temporally, superiorly, and inferiorly. Data on conjunctival surface temperature, $8 \mathrm{~mm}$ temporally, from the corneal centre were also collected (Figure 1). We chose $8 \mathrm{~mm}$ temporally from the corneal centre following a detailed examination of all collected images, as this was the location at which data could be extracted for all participants; the nasal, superior, and inferior conjunctival locations were covered by the eyelids in most of the participants.

Next, all participants underwent a complete standard ophthalmological examination. The best spectacle corrected visual acuity was determined. In addition, patients were examined using a TMS-5 corneal topographer (Tomey, Nürnberg, Germany) and a rotating Scheimpflug camera (Pentacam HR, Oculus Optikgeräte GmbH, Wetzlar, Germany). From the anterior corneal surface, we extracted the following parameters: the surface asymmetry index (SAI), surface regularity index (SRI), Klyce/Maeda keratoconus index (KCI), Smolek/Klyce keratoconus severity index (KSI), and keratoconus prediction index (KPI). From the tomographic examination, the following parameters were extracted: index of surface variance (ISV), index of surface asymmetry (IVA), keratoconus index (KI), central keratoconus index (CKI), index of height asymmetry (IHA), index of height decentration (IHD), central corneal thickness (CCT), pachymetry at the centre of the pupil (PCP), and pachymetry at the corneal apex (PCA).

A total of 179 eyes in 90 patients with KC (topographic KC classification (TKC) $0-1$ to 4 ) and 82 eyes in 41 controls 


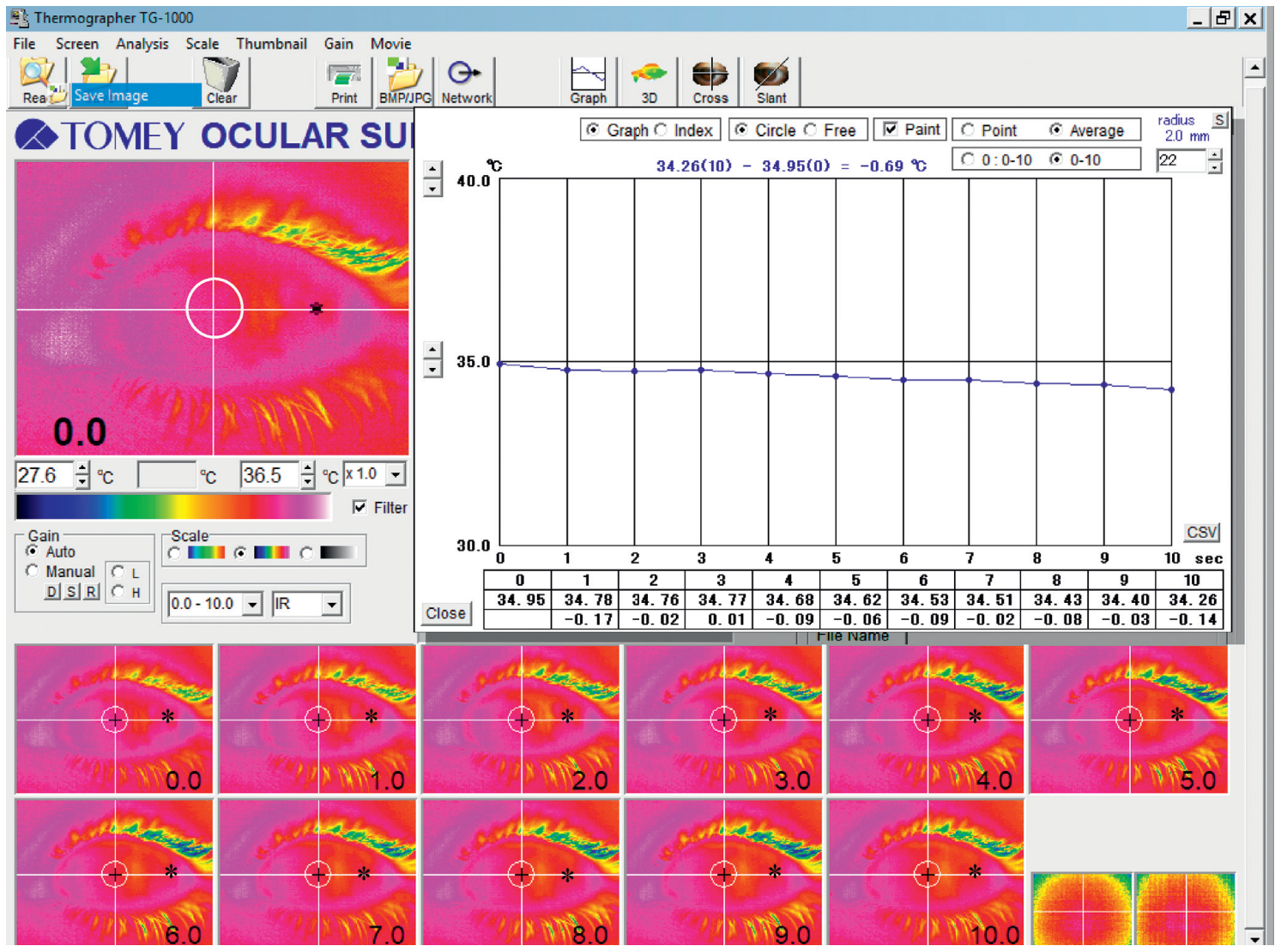

FiguRE 1: Ocular surface thermography in a keratoconus patient. We extracted mean ocular surface temperature values from the corneal centre (see $\mathrm{x}$ ) and $2 \mathrm{~mm}$ from the corneal centre nasally, temporally, superiorly, and inferiorly during 10 seconds of sustained eye opening after blinking (crossing of the white circle and the white lines). Data on ocular surface temperature conjunctivally, $8 \mathrm{~mm}$ temporally, from the corneal centre $\left({ }^{*}\right)$ were also collected.

were examined. Participants' age at the time of examination was $36.1 \pm 12.5$ (range 14-67) years in the KC group and $36.4 \pm 12.8$ (range 18-78) years in the control group $(p=0.923)$. The KC group was $34.1 \%$ females and included $53.6 \%$ left eyes, whereas the control group was $52.4 \%$ females and included $47.6 \%$ left eyes. Thirty-one (38\%) eyes in the control group ( $71 \%$ soft and $29 \%$ rigid contact lenses) and 78 (44\%) eyes (all rigid contact lenses) in the KC group had previous contact lens wear. Unfortunately, we could not gather information on the number of hours with occasional/ daily contact lens wear.

Statistical analyses were performed using SPSS software (SPSS version 19.0, IBM, New York). For the statistical analysis, both eyes were used for each participant based on the assumption that each eye and its measurements was an independent sample. Nevertheless, we have to take into account that the participants cannot discriminate between a worse and a better eye, as the questionnaire summarizes the symptoms for both eyes. Therefore, analysing the correlation of the OSDI score/subscores is always biased through analysis of the other eye in the same patient.

A test for normal distribution was performed qualitatively for both groups using the P-P plot. The Man$n$-Whitney $U$ test was then used to investigate differences in SAI, SRI, KCI, KSI, KPI, ISV, IVA, KI, CKI, IHA, IHD, OSDI scores, vision- and discomfort-related OSDI subscores, and central,/superior/inferior/nasal/conjunctival OST, CCT, PCP, and PCA between both groups. $P$ values less than 0.05 were considered significant.

The Spearman correlation test was used to analyse the interaction between OSDI, vision- and discomfort-related OSDI subscores, SAI, SRI, and OST at the corneal centre and other corneal and conjunctival regions, and CCT (all are metric variables). The Spearman correlation was also used to analyse the interaction between OST and age, the indices SAI and SRI, and CCT. The strength of the correlation was determined to be very strong $(r \geq 0.8)$, moderately strong from 0.6 to 0.8 , fair from 0.3 to 0.5 , and poor $<0.3$ [32].

\section{Results}

Best spectacle corrected visual acuity was $0.6 \pm 0.3$ in the $\mathrm{KC}$ group and $0.9 \pm 0.2$ in controls. The refractive cylinder was $-3.5 \pm 2.8 \mathrm{D}$ in the $\mathrm{KC}$ group and $-1.0 \pm 1.0 \mathrm{D}$ in controls. From corneal topography and tomography, SAI, SRI, KCI, KSI, KPI, ISV, IVA, KI, CKI, IHA, and IHD are given in Table 1 for both groups. Tables $2-3$ provide the OSDI scores and subscores, corneal and conjunctival OST values, and CCT, PCP, and PCA in both groups. We found a significant difference in SAI, SRI, KCI, KSI, KPI, ISV, IVA, KI, CKI, 
TABLE 1: Corneal topographic and tomographic data for the keratoconus patients and controls.

\begin{tabular}{lccccccccccc}
\hline & SAI & SRI & KCI & KSI & KPI & ISV & IVA & KI & CKI & IHA & IHD \\
\hline Keratoconus & $2.1 \pm 1.6$ & $1.0 \pm 0.6$ & $57.0 \pm 37.2$ & $50.3 \pm 29.4$ & $0.35 \pm 0.1$ & $84.7 \pm 49.4$ & $0.9 \pm 0.5$ & $1.2 \pm 0.2$ & $1.1 \pm 0.1$ & $26.5 \pm 22.2$ & $0.1 \pm 0.1$ \\
Control & $0.4 \pm 0.2$ & $0.2 \pm 0.2$ & $1.8 \pm 6.4$ & $2.5 \pm 8.3$ & $0.2 \pm 0.02$ & $18.16 \pm 7.6$ & $0.1 \pm 0.1$ & $1.0 \pm 0.2$ & $1.0 \pm 0.0$ & $6.0 \pm 5.5$ & $0.01 \pm 0.01$ \\
$p$ value & $<\mathbf{0 . 0 1}$ & $<\mathbf{0 . 0 1}$ & $<\mathbf{0 . 0 1}$ & $<\mathbf{0 . 0 1}$ & $<\mathbf{0 . 0 1}$ & $<\mathbf{0 . 0 1}$ & $<\mathbf{0 . 0 1}$ & $<\mathbf{0 . 0 1}$ & $<\mathbf{0 . 0 1}$ & $<\mathbf{0 . 0 1}$ & $<\mathbf{0 . 0 1}$ \\
\hline
\end{tabular}

Data are given as mean \pm SD. SAI, surface asymmetry index; SRI, surface regularity index; KCI, Klyce/Maeda keratoconus index; KSI, Smolek/Klyce neural network index; KPI, keratoconus prediction index; ISV, index of surface variance; IVA, index of vertical asymmetry; KI, keratoconus index; CKI, central keratoconus index; IHA, index of height asymmetry; IHD, index of height decentration. A significant difference was found in all factors between both groups $(p<0.01)$.

IHA, IHD, CCT, PCP, and PCA between both groups $(p<0.001)$.

The OSDI score $(31.4 \pm 22.4$ vs. $17.5 \pm 17.9)$, vision-related subscore $(17.7 \pm 14.6$ vs. $10.5 \pm 13.2)$, and discomfortrelated subscore $(14.3 \pm 10.7$ vs. $9.4 \pm 10.5)$ were significantly higher in the $\mathrm{KC}$ patients than in controls $(p<0.001)$. The average central OST was $34.2 \pm 0.6^{\circ} \mathrm{C}$ in $\mathrm{KC}$ patients and $34.2 \pm 0.6^{\circ} \mathrm{C}$ in controls $(p=0.56)$. We found no significant difference in central $\left(34.2 \pm 0.6^{\circ} \mathrm{C}\right.$ vs. $\left.34.2 \pm 0.7^{\circ} \mathrm{C}\right)$, nasal $\left(34.2 \pm 0.6^{\circ} \mathrm{C}\right.$ vs. $\left.34.2 \pm 0.7^{\circ} \mathrm{C}\right)$, temporal $\left(34.2 \pm 0.6^{\circ} \mathrm{C}\right.$ vs. $\left.34.2 \pm 0.6^{\circ} \mathrm{C}\right)$, and superior $\left(34.2 \pm 0.6^{\circ} \mathrm{C}\right.$ vs. $\left.34.2 \pm 0.6^{\circ} \mathrm{C}\right)$ OST between the two groups $(p \geq 0.22)$.

According to TKC, 24 eyes were classified as stage 1 $(13.4 \%), 55$ eyes as stage $2(30.7 \%), 51$ eyes as stage $3(28.5 \%)$, and 24 eyes as stage $4(13.4 \%)$. Patients with a TKC between two stages (e.g., TKC 0-1) were always classified as the more advanced stage. Using the Kruskal-Wallis test, we found no difference in the OST between less and more advanced stages of $\mathrm{KC}$; therefore, we did not perform a correlation analysis for the KC subgroups.

OSDI score poorly correlated with the SAI $(r=0.295$, $p<0.001)$ and fairly correlated with the SRI $(r=0.354$, $p<0.001)$, but did not correlate with OST at the corneal centre $(r=-0.012)$ or other corneal or conjunctival regions $(r \geq-0.072)$. OSDI also did not correlate with CCT in either group $(r=-0.270)$.

The correlation of the vision- and discomfort-related OSDI subscores with SAI, SRI, and OST at the corneal centre in different stages of $\mathrm{KC}$ are shown in Table 4. For all participants, vision- and discomfort-related OSDI subscores poorly to fairly correlated with SRI and SAI $(r>0.174$, $p<0.005)$, but none of the subscores correlated with OST $(r<0.001)$. In some of the subgroups (control, KC1, and $\mathrm{KC} 2$ ), the subscores correlated poorly with SAI and SRI, and the discomfort-related OSDI subscore poorly correlated with OST (Table 4).

OST at all of the examined regions also fairly correlated with patient age $(-0.177 \geq r \geq-0.310)$ in the KC group and did not correlate with the control group $(-0.10 \geq r \geq-0.074)$. OST at the corneal centre also did not correlate with the SAI $(r=-0.056)$, SRI $(r=-0.086)$, or CCT $(r=0.048)$.

\section{Discussion}

The most conspicuous finding of our study is that OST does not differ between $\mathrm{KC}$ patients and controls, though the OSDI score was significantly higher in KC patients than in controls. In addition, OST at the corneal centre did not correlate with SAI, SRI, TKC ( $p \geq 0.18)$, CCT, PCP, or PCA $(p \geq 0.06)$. The OSDI score and vision- and discomfort-related OSDI subscores poorly to fairly correlated with the SAI and SRI, but did not correlate with central corneal OST.

The aetiology of KC remains unclear. However, several authors have discussed a potential inflammatory cofactor [22-24]. Allergic conjunctivitis and dry eye syndrome are very common among $\mathrm{KC}$ patients $[21,26]$. In patients with dry eye, the OST is increased, and OST decreases quicker during sustained eye opening than in healthy adults [7, 32-34]. Moussa et al. [35] could not find any diurnal changes in the OST of healthy adults. Morgan et al. [8] also found an increase in the OST throughout the day, especially in dry eyes. These findings suggest that diurnal changes in OST indicate ocular surface abnormalities or corneal pathology. Analysing the diurnal changes in the OST of KC patients was not the aim of the present study, but it could be interesting to assess the diurnal variations in OST in $\mathrm{KC}$ patients in the future.

Hara et al. found a significant correlation between the conjunctival surface temperature and the severity of conjunctival allergic disease, and OST was a useful measure to determine the effectiveness of antiallergy agents [36]. The increase in the OSDI score in KC patients may reflect dry eye disease or could be related to the poor visual outcomes in $\mathrm{KC}$ patients. With an increase in both the vision- and discomfort-related OSDI subscores in $\mathrm{KC}$, we could determine that both ocular surface disease and deteriorated visual acuity contribute to the increased OSDI score. However, this is not mirrored by an increase in the OST. Data on conjunctival allergic disease were not collected in the present study.

Corneal innervation may also play a decisive role in OST. The cornea is densely innervated by the fibres of the ophthalmic branch of the trigeminus nerve, known as ciliary nerves [37]. Corneal nerves are important for corneal homeostasis due to their protective functions and their role in wound healing and in regulating corneal sensation [38, 39]. Epithelial dendritic cells (Langerhans cells) are inflammatory, antigen-presenting corneal cells responsible for immune surveillance. These cells are distributed from the basal epithelial corneal layer to the sub-basal nerve plexus [40]. Mature Langerhans cell morphology is frequently seen in the periphery of the cornea, whereas immature cells are seen centrally [41].

The sub-basal nerve plexus and the epithelial dendritic cell density have been examined in different subtypes of dry eye disease. Tepelus et al. [42] found a reduction in the sub- 


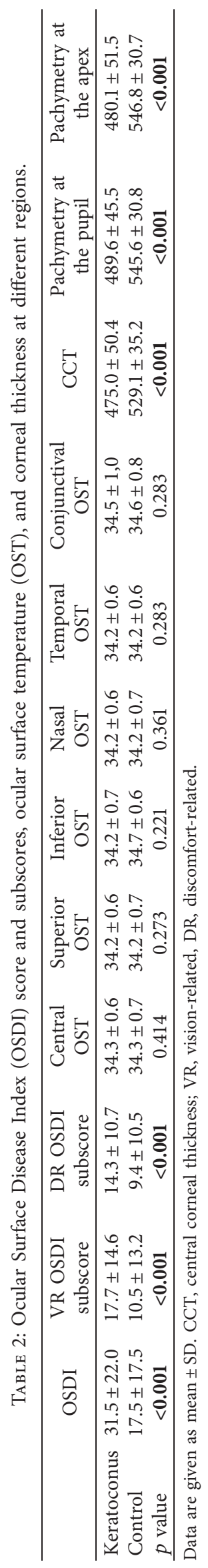


TABLE 3: Ocular Surface Disease Index (OSDI) score and subscores and central and conjunctival ocular surface temperature (OST) in different stages of keratoconus $(\mathrm{KC})$ and controls.

\begin{tabular}{lccccc}
\hline & OSDI & VR subscore & DR subscore & Central OST & Conjunctival OST \\
\hline KC4 & $41.9 \pm 25.9$ & $25.6 \pm 18.0$ & $17.5 \pm 12.5$ & $34.2 \pm 0.6$ & $34.5 \pm 0.9$ \\
KC3 & $29.3 \pm 18.5$ & $16.3 \pm 11.9$ & $13.0 \pm 9.7$ & $34.3 \pm 0.7$ & $34.5 \pm 1.2$ \\
KC2 & $31.7 \pm 25.1$ & $17.6 \pm 15.9$ & $14.8 \pm 12.4$ & $34.2 \pm 0.6$ & $34.5 \pm 0.9$ \\
KC1 & $30.1 \pm 19.2$ & $15.4 \pm 11.5$ & $14.0 \pm 8.9$ & $34.3 \pm 0.7$ & $34.3 \pm 1.0$ \\
Control & $17.5 \pm 17.5$ & $10.5 \pm 13.2$ & $9.4 \pm 10.5$ & $34.3 \pm 0.7$ & $34.6 \pm 0.8$ \\
\hline
\end{tabular}

Data are given as mean \pm SD. VR, vision-related; DR, discomfort-related.

TABLE 4: Spearman correlation of vision- and discomfort-related Ocular Surface Disease Index (OSDI) subscores with surface characteristics at the corneal centre in different stages of keratoconus (KC) and controls.

\begin{tabular}{lccc}
\hline & SAI & SRI & Central OST \\
\hline KC4-VR OSDI & -0.29 & -0.08 & -0.18 \\
KC3-VR OSDI & -0.12 & -0.05 & -0.007 \\
KC2-VR OSDI & -0.07 & $0.26 p=0.04$ & 0.03 \\
KC1-VR OSDI & 0.33 & 0.02 & 0.35 \\
Control-VR OSDI & $0.22 p=0.03$ & $0.32 p=0.002$ & -0.07 \\
KC4-DR OSDI & -0.18 & 0.24 & -0.09 \\
KC3-DR OSDI & -0.27 & -0.19 & -0.04 \\
KC2-DR OSDI & -0.15 & 0.18 & 0.02 \\
KC1-DR OSDI & 0.27 & 0.18 & $0.45 p=0.02$ \\
Control-DR OSDI & $0.26 p=0.01$ & $0.25 p=0.01$ & -0.01 \\
\hline
\end{tabular}

VR, vision-related; DR, discomfort-related; SAI, surface asymmetry index; SRI, surface regularity index; OST, ocular surface temperature. $r$ values are given, with $p$ values shown in the case of significance.

basal nerve plexus and an increase in inflammatory dendritic cell density in Sjögren and non-Sjögren dry eye subgroups [43]. Many studies have demonstrated abnormal corneal nerve morphology and branching patterns, reduced nerve density, increased tortuosity, and thickening in KC [44, 45]. Mandathara et al. found mature Langerhans cells at the centre of the cornea, which also supports an inflammatory origin of KC [46]. To the best of our knowledge, the relationship between changes in the sub-basal nerve plexus and Langerhans cell density and OST has not yet been analysed.

The literature offers controversial information on the effect of corneal thickness on OST. Morgan reported a significant decrease in OST with increasing corneal thickness [47]. In contrast, Alio and Padron [48] and Efron et al. [49] found a progressive increase in OST from the corneal centre to the periphery. Purslow and Wolffsohn [50] found a weak negative correlation between corneal thickness and OST using the Thermo Tracer 7210MX. Pattmöller et al. [51] could not verify a correlation between local corneal thickness and local OST at any point on the corneal surface in healthy adults. The relation of the OST and the anterior chamber depth is also contraversial $[48,49,51]$. In the present study, we could not determine a correlation between corneal thickness and OST in KC patients or controls.

In summary, we found a significantly increased OSDI score in KC patients compared to an age-matched control group. However, this was not accompanied by an increase in the OST at any stage of KC. We could not clarify whether the reduced corneal thickness in $\mathrm{KC}$ patients may have a "corneal-cooling effect". Our study also shows that both vision- and discomfort-related symptoms of $\mathrm{KC}$ have to be managed in parallel in ophthalmologic practice, but the necessity of anti-inflammatory treatment cannot be verified through ocular thermography.

\section{Data Availability}

The data used to support the findings of this study are included within the article.

\section{Disclosure}

The views expressed in the submitted article are the authors' own and not an official position of the institution or funder.

\section{Conflicts of Interest}

The authors declare no conflicts of interest related to this study.

\section{Acknowledgments}

The work of Dr. Németh at the Department of Ophthalmology of Saarland University Medical Center was supported by a grant from the European Board of Ophthalmology.

\section{References}

[1] J. R. Harding, "Investigating deep venous thrombosis with infrared imaging," IEEE Engineering in Medicine and Biology Magazine, vol. 17, no. 4, pp. 43-46, 1998.

[2] R. Hatwar and C. Herman, "Inverse method for quantitative characterisation of breast tumours from surface temperature data," International Journal of Hyperthermia, vol. 33, pp. 741-757, 2017.

[3] R. Lasanen, E. Piippo-Savolainen, T. Remes-Pakarinen et al., "Thermal imaging in screening of joint inflammation and rheumatoid arthritis in children," Physiological Measurement, vol. 36, no. 2, pp. 273-282, 2015.

[4] R. Mapstone, "Ocular thermography," British Journal of Ophthalmology, vol. 54, no. 11, pp. 751-754, 1970.

[5] R. Mapstone, "Corneal thermal patterns in anterior uveitis," British Journal of Ophthalmology, vol. 52, no. 12, pp. 917-921, 1968.

[6] R. Mapstone, "Determinants of corneal temperature," British Journal of Ophthalmology, vol. 52, no. 10, pp. 729-741, 1968.

[7] A. A. Kawali, "Thermography in ocular inflammation," Indian Journal of Radiology and Imaging, vol. 23, pp. 281-283, 2013. 
[8] P. B. Morgan, A. B. Tullo, and N. Efron, "Infrared thermography of the tear film in dry eye," Eye, vol. 9, no. 5, pp. 615-618, 1995.

[9] S. Kawasaki, S. Mizoue, M. Yamaguchi et al., "Evaluation of filtering bleb function by thermography," British Journal of Ophthalmology, vol. 93, no. 10, pp. 1331-1336, 2009.

[10] S. Betney, P. B. Morgan, S. J. Doyle et al., "Corneal temperature changes during photorefractive keratectomy," Cornea, vol. 16, pp. 158-161, 1997.

[11] A. Belkin, A. Abulafia, A. Michaeli et al., "Wound temperature profiles of coaxial mini-incision versus sleeveless microincision phacoemulsification," Clinical \& Experimental Ophthalmology, vol. 45, pp. 247-253, 2017.

[12] K. Konieczka, A. Schoetzau, S. Koch, D. Hauenstein, and J. Flammer, "Cornea thermography: optimal evaluation of the outcome and the resulting reproducibility," Translational Vision Science \& Technology, vol. 7, no. 3, p. 14, 2018.

[13] M. H. Geiser, M. Bonvin, and O. Quibel, "Corneal and retinal temperatures under various ambient conditions: a model and experimental approach," Klinische Monatsblätter für Augenheilkunde, vol. 221, no. 5, pp. 311-314, 2004.

[14] L. Kessel, L. Johnson, and H. Arvidsson, "The relationship between body and ambient temperature and corneal temperature," Investigative Opthalmology \& Visual Science, vol. 51, no. 12, pp. 6593-6597, 2010.

[15] R. D. Larsen and I. Fatt, "Environmental influences on ocular temperature," Investigative Ophthalmology \& Visual Science, vol. 12, pp. 596-602, 1973.

[16] J. K. Slettedal and A. Ringvold, "Correlation between corneal and ambient temperature with particular focus on polar conditions," Acta Ophthalmologica, vol. 93, no. 5, pp. 422426, 2015.

[17] C. Purslow and J. S. Wolffsohn, "Ocular surface temperature," Eye \& Contact Lens: Science \& Clinical Practice, vol. 31, no. 3, pp. 117-123, 2005.

[18] K. Gugleta, S. Orgül, and J. Flammer, "Is corneal temperature correlated with blood-flow velocity in the ophthalmic artery?," Current Eye Research, vol. 19, no. 6, pp. 496-501, 1999.

[19] Y. S. Rabinowitz, "Keratoconus," Survey of Ophthalmology, vol. 42, no. 4, pp. 297-319, 1998.

[20] H.-Y. P. Chang and J. Chodosh, "The genetics of keratoconus," Seminars in Ophthalmology, vol. 28, no. 5-6, pp. 275280, 2013.

[21] K. H. Weed, C. J. Macewen, T. Giles, and C. N. J. Low, "The Dundee University Scottish Keratoconus study: demographics, corneal signs, associated diseases, and eye rubbing," Eye, vol. 22, no. 4, pp. 534-541, 2008.

[22] I. Lema, T. Sobrino, J. A. Duran et al., "Subclinical keratoconus and inflammatory molecules from tears," British Journal of Ophthalmology, vol. 93, no. 6, pp. 820-824, 2009.

[23] B. L. Kolozsvári, G. Petrovski, P. Gogolák et al., “Association between mediators in the tear fluid and the severity of keratoconus," Ophthalmic Research, vol. 51, no. 1, pp. 46-51, 2014.

[24] D. Pásztor, B. L. Kolozsvári, A. Csutak et al., "Scheimpflug imaging parameters associated with tear mediators and bronchial asthma in keratoconus," Journal of Ophthalmology, vol. 2016, Article ID 9392640, 7 pages, 2016.

[25] M. Dogru, H. Karakaya, H. Özçetin et al., "Tear function and ocular surface changes in keratoconus," Ophthalmology, vol. 110, no. 6, pp. 1110-1118, 2003.

[26] C. S. De Paiva, L. D. Harris, and S. C. Pflugfelder, "Keratoconus-like topographic changes in keratoconjunctivitis sicca," Cornea, vol. 22, no. 1, pp. 22-24, 2003.
[27] E. Zemova, T. Eppig, B. Seitz et al., "Interaction between topographic/tomographic parameters and dry eye disease in keratoconus patients," Current Eye Research, vol. 39, no. 1, pp. 1-8, 2014.

[28] S. Goebels, B. Seitz, and A. Langenbucher, "Diagnostics and stage-oriented therapy of keratoconus: introduction to the Homburg keratoconus center (HKC)," Der Ophthalmologe, vol. 110, no. 9, pp. 808-809, 2013.

[29] P. M. Mathews, P. Y. Ramulu, D. S. Friedman, and E. K. Utine, "Evaluation of ocular surface disease in patients with glaucoma," Ophthalmology, vol. 120, no. 11, pp. 2241-2248, 2013.

[30] M. Pattmöller, J. Wang, J. Pattmöller et al., "Inter-und Intraobserverreliabilität der kornealen Oberflächentemperaturmessung mit dem TG-1000 bei Normalaugen," Der Ophthalmologe, vol. 112, no. 9, pp. 746-751, 2015.

[31] A. Mori, Y. Oguchi, Y. Okusawa et al., "Use of high-speed, high-resolution thermography to evaluate the tear film layer," American Journal of Ophthalmology, vol. 124, no. 6, pp. 729-735, 1997.

[32] Y. H. Chan, "Biostatistics 104: correlational analysis," Singapore Medical Journal, vol. 44, pp. 614-619, 2003.

[33] M. K. J. Klamann, A.-K. B. Maier, J. Gonnermann, J. P. Klein, E. Klein, and U. Pleyer, "Ocular surface temperature gradient is increased in eyes with bacterial corneal ulcers," Ophthalmic Research, vol. 49, no. 1, pp. 52-56, 2013.

[34] T. Kamao, M. Yamaguchi, S. Kawasaki, S. Mizoue, A. Mizoue, and Y. Ohashi, "Screening for dry eye with newly developed ocular surface thermographer," American Journal of Ophthalmology, vol. 151, no. 5, pp. 782-791, 2011.

[35] S. Moussa, T. Eppig, J. Pattmöller et al., "Diurnal and zonal analysis of corneal surface temperature in young healthy adults," European Journal of Ophthalmology, vol. 23, no. 5, pp. 641-645, 2013.

[36] Y. Hara, A. Shiraishi, M. Yamaguchi, T. Kawasaki, and Y. Ohashi, "Evaluation of allergic conjunctivitis by thermography," Ophthalmic Research, vol. 51, no. 3, pp. 161-166, 2014.

[37] L. J. Müller, L. Pels, and G. F. Vrensen, "Ultrastructural organization of human corneal nerves," Investigative Ophthalmology \& Visual Science, vol. 37, p. 476, 1996.

[38] R. F. Guthoff, H. Wienss, C. Hahnel, and A. Wree, "Epithelial innervation of human cornea: a three-dimensional study using confocal laser scanning fluorescence microscopy," Cornea, vol. 24, no. 5, pp. 608-613, 2005.

[39] L. Wree and N. Efron, "Morphology of corneal nerve using confocal microscopy," Cornea, vol. 20, no. 4, pp. 374-384, 2001.

[40] A. Zhivov, J. Stave, B. Vollmar, and R. Guthoff, "In vivo confocal microscopic evaluation of Langerhans cell density and distribution in the normal human corneal epithelium," Graefe's Archive for Clinical and Experimental Ophthalmology, vol. 243, no. 10, pp. 1056-1061, 2005.

[41] A. Zhivov, J. Stave, B. Vollmar, and R. Guthoff, "In vivo confocal microscopic evaluation of Langerhans cell density and distribution in the corneal epithelium of healthy volunteers and contact lens wearers," Cornea, vol. 26, no. 1, pp. 47-54, 2007.

[42] T. C. Tepelus, G. B. Chiu, J. Huang et al., "Correlation between corneal innervation and inflammation evaluated with confocal microscopy and symptomatology in patients with dry eye syndromes: a preliminary study," Graefe's Archive for Clinical and Experimental Ophthalmology, vol. 255, no. 9, pp. 1771-1778, 2017.

[43] I. K. Gipson, P. Argüeso, R. Beuerman et al., "Research in dry eye: report of the research Subcommittee of the International 
dry eye workshop," The Ocular Surface, vol. 5, pp. 179-193, 2007.

[44] m. r. U??akhan, A. Kanpolat, N. 1. Ylmaz et al., "In vivo confocal microscopy findings in keratoconus," Eye \& Contact Lens: Science \& Clinical Practice, vol. 32, no. 4, pp. 183-191, 2006.

[45] G. ??zkan, A. Ozkagnici, B. Bozkurt, and R. A. Malik, "In vivo corneal confocal microscopic analysis in patients with keratoconus," International Journal of Ophthalmology, vol. 18, no. 8, pp. 534-539, 2015.

[46] P. S. Mandathara, F. J. Stapleton, J. Kokkinakis et al., “A pilot study on corneal Langerhans cells in keratoconus," Contact Lens and Anterior Eye, vol. 41, no. 2, pp. 219-223, 2018.

[47] P. Willcox, Ocular thermography in health and disease, Ph.D. thesis, University of Manchester Institute of Science and Technology, Manchester, England, 1994.

[48] J. Aliò and M. Padron, "Influence of age on the temperature of the anterior segment of the eye," Ophthalmic Research, vol. 14, no. 3, pp. 153-159, 1982.

[49] N. Efron, G. Young, and N. A. Brennan, "Ocular surface temperature," Current Eye Research, vol. 8, pp. 901-906, 1989.

[50] C. Purslow and J. Wolffsohn, "The relation between physical properties of the anterior eye and ocular surface temperature," Optometry and Vision Science, vol. 84, no. 3, pp. 197-201, 2007.

[51] J. Pattmöller, J. Wang, E. Zemova et al., "Correlation of corneal thickness, endothelial cell density and anterior chamber depth with ocular surface temperature in normal subjects," Zeitschrift für Medizinische Physik, vol. 25, no. 3, pp. 243-250, 2015. 